\title{
Size Dependent Optical Nonlinearity and Optical Limiting Properties of Water Soluble CdSe Quantum Dots
}

\author{
Anju K. Augustine, S. Mathew, P. Radhakrishnan, V. P. N. Nampoori, and M. Kailasnath \\ International School of Photonics, Cochin University of Science and Technology, Cochin, Kerala 682022, India \\ Correspondence should be addressed to Anju K. Augustine; srrosmin2009@gmail.com
}

Received 4 July 2014; Accepted 4 November 2014; Published 26 November 2014

Academic Editor: Fengqiang Sun

Copyright (C) 2014 Anju K. Augustine et al. This is an open access article distributed under the Creative Commons Attribution License, which permits unrestricted use, distribution, and reproduction in any medium, provided the original work is properly cited.

\begin{abstract}
We present third-order optical nonlinear absorption in CdSe quantum dots (QDs) with particle sizes in the range of 4.16-5.25 nm which has been evaluated by the $Z$-scan technique. At an excitation irradiance of $0.54 \mathrm{GW} / \mathrm{cm}^{2}$ the CdSe QDs exhibit reverse saturation indicating a clear nonlinear behavior. Nonlinearity increases with particle size in CdSe QDs within the range of our investigations which in turn depends on the optical band gap. The optical limiting threshold of the QDs varies from $0.35 \mathrm{GW} / \mathrm{cm}^{2}$ to $0.57 \mathrm{GW} / \mathrm{cm}^{2}$ which makes CdSe QDs a promising candidate for reverse-saturable absorption based devices at high laser intensities such as optical limiters.
\end{abstract}

\section{Introduction}

Before the advent of lasers, transparent optical materials were assumed to be essentially passive unaffected by the light travelling through them. The high powers of laser beams made it possible to observe that the effect of light on a medium can indeed change its properties such as refractive index or absorption. These are optical nonlinear phenomena. When this happens, the light itself gets affected by this change in a nonlinear way; for example, the nonlinear response of the material can convert the laser light into new colours, both harmonics of the optical frequency and sum and difference frequencies. With the development of optical communication networks, various nonlinear optical (NLO) devices such as optical switches, optical limiters, optical detectors, and optical sensors have attracted considerable attention because of their widespread usage for scientific and industrial purposes. Among all the NLO properties, optical limiting is one of the most promising practical applications, as it can protect the human eye and photosensitive components from damage caused by intense optical radiation $[1,2]$. Optical limiting results from irradiance-dependent NLO responses of materials in which the incoming intense light alters the refractive and absorptive properties, resulting in a greatly reduced transmitted intensity. It is important to select suitable materials as optical limiting media by determining the magnitude of their nonlinearity. Organic materials characterized by large NLO responses are of major interest owing to their large NLO susceptibilities, fast response time, architectural flexibility, low cost, and ease of fabrication $[3,4]$.

In recent years, interest in the synthesis, characterization, and application of colloidal quantum dot (QD) semiconductor materials has grown markedly. QDs of cadmium selenide (CdSe) are by far the most studied system among all the semiconducting nanocrystals. The bulk CdSe has a direct band gap of $1.74 \mathrm{eV}$ at $300 \mathrm{~K}$ and a typical Bohr exciton diameter of around $5.6 \mathrm{~nm}$ [5]. Consequently, CdSe QDs show sizable quantum confinement effects with remarkably different optical properties. The size dependent, unusual optical and electronic properties of these QDs have been studied in detail using a wide variety of experimental and theoretical techniques. The linear optical properties of CdSe QDs depend strongly on particle size; for example, the blue shift of excitonic absorption and emission peaks with decreasing particle size is a well-known observation. The unusual properties of the QDs, in the quantum-confined regime, have led to numerous technological applications.

In the past decade, there has been increasing interest in the luminescent and nonlinear optical properties of these nanometer-sized QDs. Large optical nonlinearities in CdSe QDs have been reported using different techniques. These techniques include degenerate four-wave mixing and $Z$-scan 


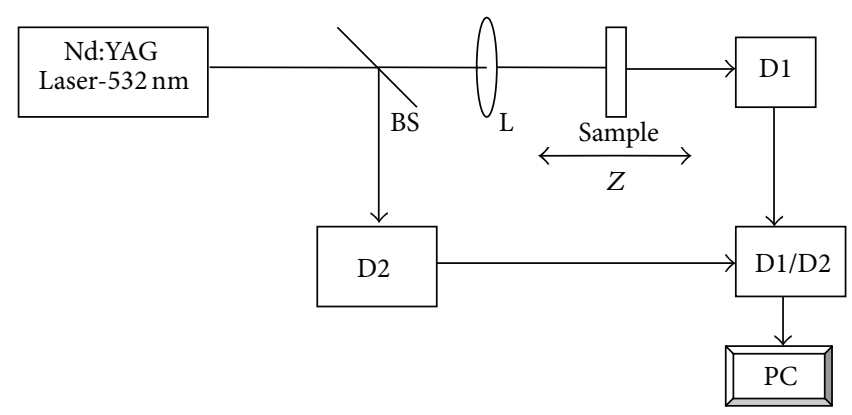

BS: Beam splitter

L: Lens

D1, D2: Detectors

Figure 1: $Z$-scan experimental setup.

techniques with nanosecond and picosecond laser pulses. $Z$ scan, especially, is an effective technique to investigate nonlinear absorption and nonlinear refraction. An ideal optical limiter, by definition, is a device that exhibits a linear transmittance below a threshold and clamps the output to a constant above it, thus providing safety to sensors and the eyes. A wide range of materials contributing to the optical limiting and nonlinear absorption has been investigated. It is well known that optical limiting devices rely on one or more of the nonlinear optical mechanisms such as excited state absorption, free carrier absorption, two-photon absorption (TPA), thermal defocusing/scattering, photorefraction, nonlinear refraction, and induced scattering with enhancement in limiting performance by coupling two or more of such mechanisms [6-8].

In this paper, we present the study of the effect of size on the nonlinear optical properties of CdSe QDs and estimate the nonlinear absorption coefficient $\beta$ of CdSe QDs at different particle size at $532 \mathrm{~nm}$. These CdSe QDs exhibit reverse saturable absorption (RSA), in which the excited state absorption cross section is higher than the ground state absorption cross section. The origin of the third-order nonlinearity predicted from $Z$-scan studies is attributed to resonant two-photon absorption (TPA). We also report the limiting threshold as a function of the particle size of CdSe QDs.

\section{Experimental}

In the present work, CdSe NPs were prepared by a modified aqueous method using reflux $[9,10]$. The precursors used are $\mathrm{CdCl}_{2}: \mathrm{H}_{2} \mathrm{O}$ and $\mathrm{Na}_{2} \mathrm{SeO}_{3}$. The capping agent used was mercaptosuccinic acid (MSA). The molar ratio of the precursors and MSA is $4: 1: 12$, respectively. The buffer solution [11] with $\mathrm{pH} 7$ is prepared with sodium borate and citric acid in water. The precursors were added to the buffer with constant stirring. The $\mathrm{pH}$ of the corresponding colourless solution is adjusted by adding $1 \mathrm{M} \mathrm{NaOH}$. It was placed in a three-necked flask followed by reflux at $100^{\circ} \mathrm{C}$ in open-air conditions. Reflux process gives the required thermal energy to the reaction. The colour of the solution turned to bright yellow after a few minutes of reflux. The temperature of the reaction system was maintained at $100^{\circ} \mathrm{C}$. During the reflux, the colour of solution gradually changed and NPs started to form and aliquots were taken out of the flask at different times for the optical measurements to monitor the growth of the QDs. The particles tend to form agglomerates due to the strong interaction of the carboxylic groups [11] leading to a possible overestimation of the mean size distribution.

Absorption spectra of the prepared CdSe QDs were recorded using UV-Visible Spectrophotometer (Jasco V-570 UV/VIS/IR). Fluorescence spectra of QDs were obtained on a Cary Eclipse Fluorescence Spectrophotometer (Varian). The excitation wavelength was kept at $390 \mathrm{~nm}$, and the emission spectra were recorded. All absorption and fluorescence spectra were measured without any postpreparative size separation. The structural properties of the samples were investigated by X-ray diffraction (XRD) on a Bruker AXS D8 Advance X-ray diffractometer with Ni-filtered $\mathrm{Cu} \mathrm{K} \alpha$ (1.5406 $\AA$ ) source. The electron micrograph images of CdSe QDs in colloidal form were taken using transmission electron microscope (Philips Tecnai G2 at $120 \mathrm{kV}$ ). The FT-IR spectra of the samples were recorded using Thermo NICOLET 380 FTIR Spectrometer by means of $\mathrm{KBr}$ pellet procedure. Nonlinear absorption coefficient $(\beta)$ and optical limiting threshold of the samples were calculated using open aperture $Z$-scan technique employing an Nd-YAG laser (532 nm, $7 \mathrm{ns,}$ $10 \mathrm{~Hz}$ ). The experimental setup is shown in Figure 1.

\section{Results and Discussion}

For detailed study, we used samples taken after reflux times of 10 minutes, 1 hour, and 3 hours and they are denoted by $\mathrm{C}_{1}$, $\mathrm{C}_{2}$, and $\mathrm{C}_{3}$, respectively. The variation in absorption peak and the corresponding optical energy band gap (inset) of samples $\left(\mathrm{C}_{1}, \mathrm{C}_{2}\right.$, and $\left.\mathrm{C}_{3}\right)$ are shown in Figures $2(\mathrm{a}), 2(\mathrm{~b})$, and 2(c).

Energy band gap of the QDs shows a variation from $2.51 \mathrm{eV}$ to $2.34 \mathrm{eV}$ as the particle size increased during the reflux time from 10 minutes to 3 hours. It is observed that as particle size increases, absorption peak shifts to higher wavelength side and the band gap is enhanced with decrease in particle size. The emission spectra of the $C_{1}, C_{2}$, and $C_{3}$ samples are also shown in Figure 3. 


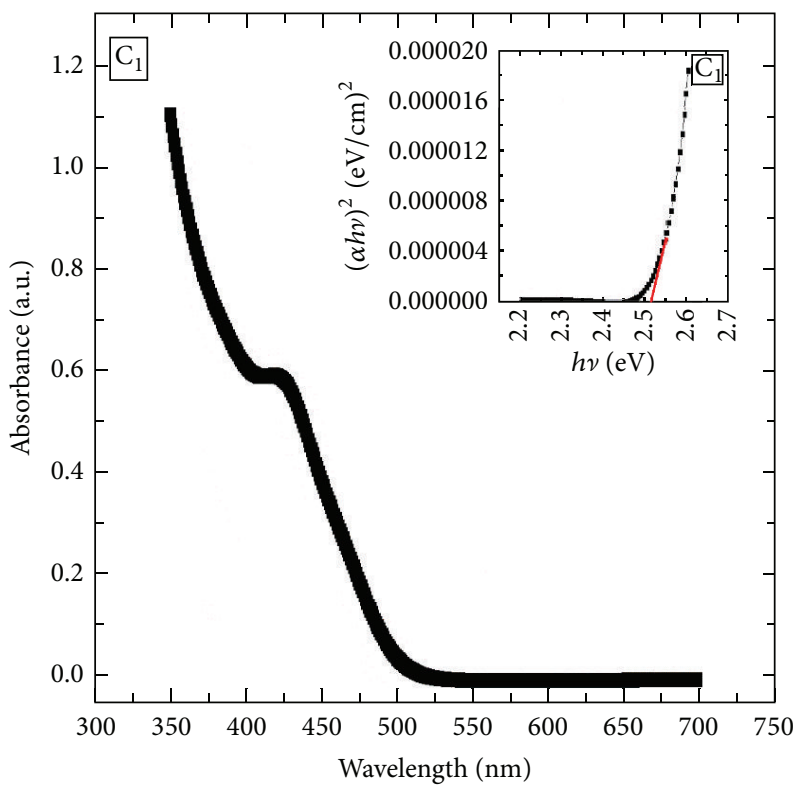

(a)

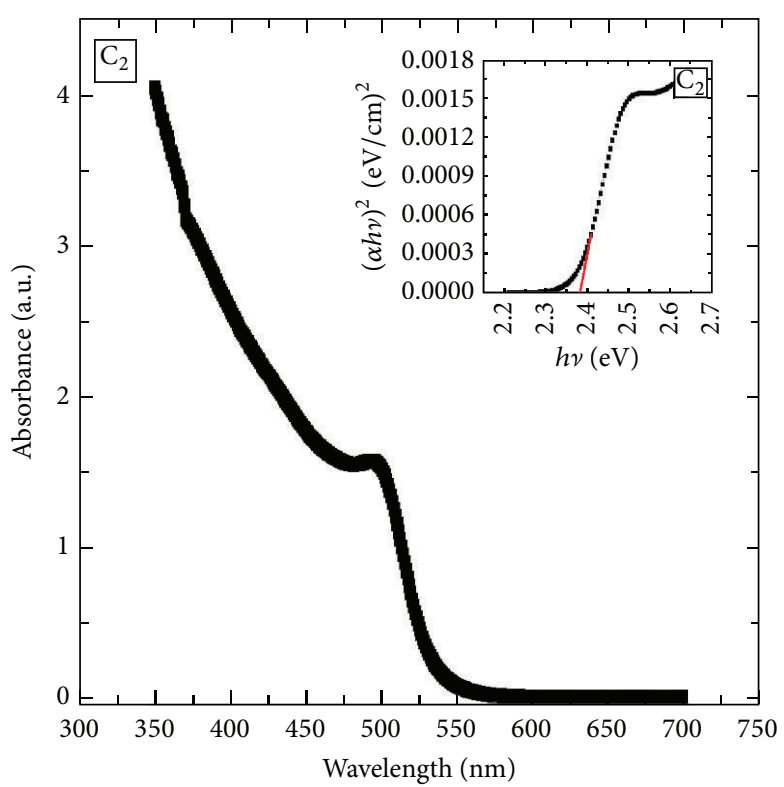

(b)

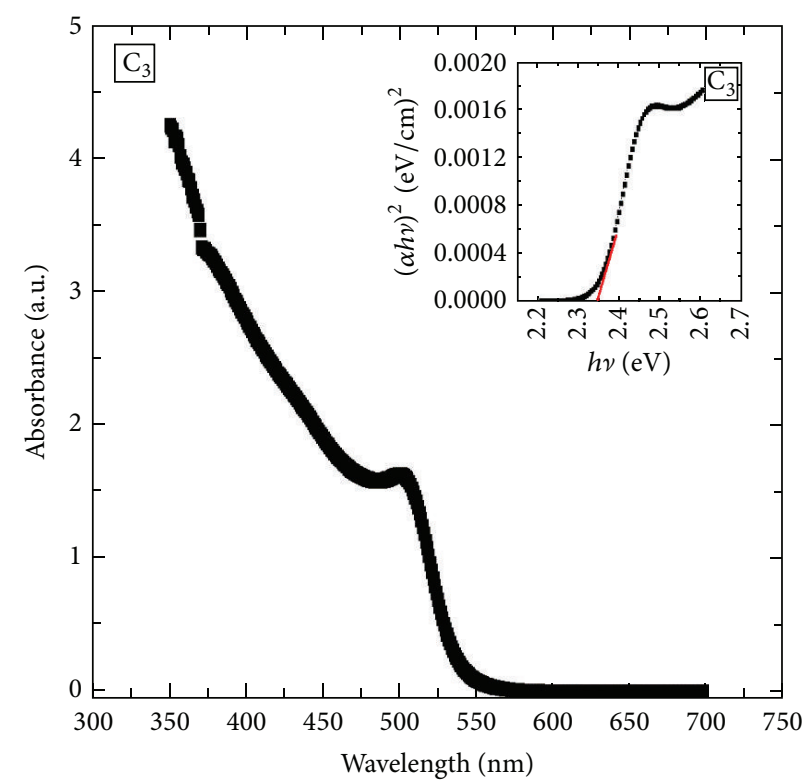

(c)

FIGURE 2: ((a), (b), and (c)) The variation in absorption peak and the corresponding optical energy band gap (inset) of samples $\left(\mathrm{C}_{1}\right.$, $\mathrm{C}_{2}$, and $\mathrm{C}_{3}$ ).

The particle size is calculated with effective mass approximation [12]. For CdSe, $E_{g}^{\text {bulk }}=1.74 \mathrm{eV}, \epsilon=10.6, m_{e}^{*}=0.13$, $m_{h}^{*}=0.45$ :

$$
E_{g}^{\text {nano }}=E_{g}^{\text {bulk }}+\frac{h^{2}}{2 m_{0} d^{2}}\left(\frac{1}{m_{e}^{*}}+\frac{1}{m_{h}^{*}}\right)-\frac{36 e^{2}}{4 \pi \epsilon \epsilon_{0} d},
$$

where $d$ is the particle diameter. The calculated particle size of these QDs is presented in Table 1. A typical XRD spectrum of CdSe QDs is shown in Figure 4 and the average particle size $(d)$ can be measured using Scherrer equation $d=$ $0.9 \lambda /(B \cos \theta)$, where $B$ is the full width at half maximum of the peak.

The calculated average particle size is about $4 \mathrm{~nm}$ for the powdered sample. The peak observed at $2 \theta(\mathrm{hkl})$ values of $27^{\circ}$ (101), $35^{\circ}(102), 41^{\circ}(110), 50^{\circ}(201)$, and $55^{\circ}$ (202) planes shows the existence of CdSe hexagonal phase (JCPDS file no. 01-0712554).

Figure 5(a) shows histogram and TEM image of $\mathrm{C}_{3}$ sample with average particle size of $4.09 \mathrm{~nm}$. It shows that the particles formed are in spherical shape and average particle size is calculated to be $4.09 \mathrm{~nm}$. Figure 5(b) represents HRTEM image of $\mathrm{C}_{3}$ sample which suggests that the prepared 
TABLE 1: Data showing particle size, energy band gap, nonlinear absorption coefficient $(\beta)$, optical limiting threshold, and imaginary part of susceptibility $\left(\operatorname{Im} \chi^{(3)}\right)$ of the samples $\left(\mathrm{C}_{1}, \mathrm{C}_{2}\right.$, and $\left.\mathrm{C}_{3}\right)$.

\begin{tabular}{lccccc}
\hline Sample & Particle size $(\mathrm{nm})$ & Energy B.G $(\mathrm{eV})$ & $\beta(\mathrm{m} / \mathrm{W})$ & Optical limiting threshold $\left(\mathrm{GW} / \mathrm{cm}^{2}\right)$ & $\mathrm{Im} \chi^{(3)} \times 10^{-11}(\mathrm{e} . \mathrm{s} . \mathrm{u})$ \\
\hline $\mathrm{C}_{1}$ & 4.17 & 2.52 & $0.27 \times 10^{-10}$ & 0.57 & 0.16 \\
$\mathrm{C}_{2}$ & 4.96 & 2.38 & $0.69 \times 10^{-10}$ & 0.48 & 0.41 \\
$\mathrm{C}_{3}$ & 5.25 & 2.34 & $2.47 \times 10^{-10}$ & 0.35 & 1.45 \\
\hline
\end{tabular}

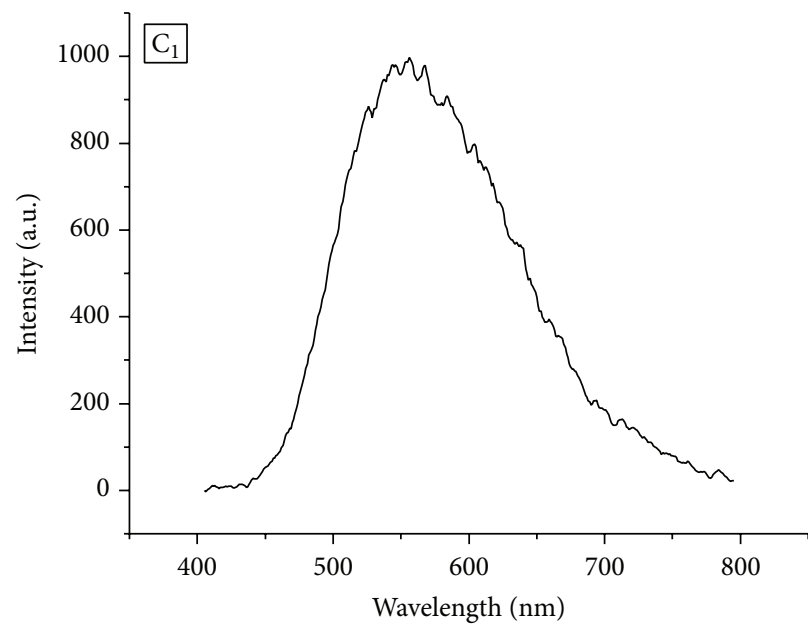

(a)

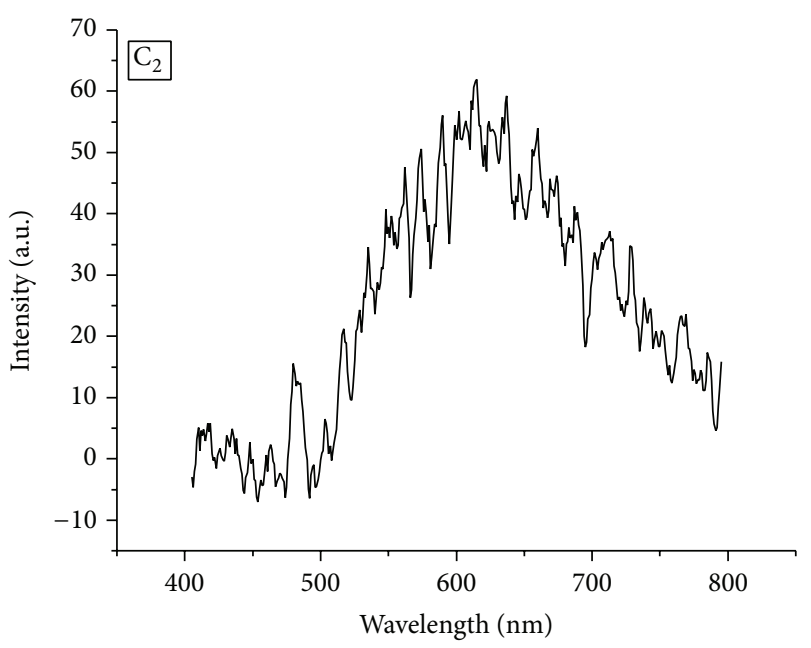

(b)

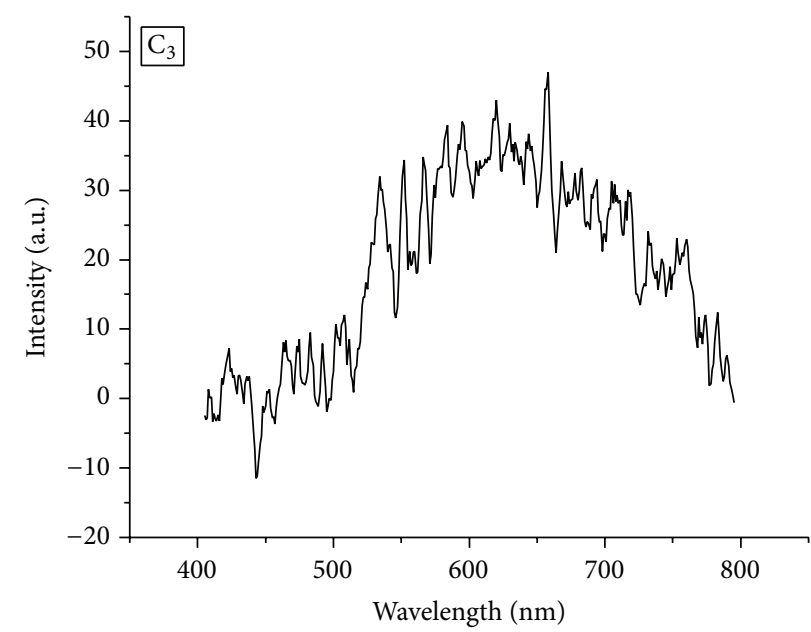

(c)

Figure 3: The emission spectra of $\mathrm{C}_{1}, \mathrm{C}_{2}$, and $\mathrm{C}_{3}$ samples under the excitation wavelength of $390 \mathrm{~nm}$.

CdSe QDs have good crystallinity and this may enable them to possess high-quality optical property.

FTIR spectrum of the $\mathrm{C}_{3}$ sample is shown in Figure 6. A broad peak at $3430 \mathrm{~cm}^{-1}$ is assigned to $-\mathrm{OH}$ stretching. The $\mathrm{Cd}-\mathrm{Se}$ band stretching can be observed at $\sim 843 \mathrm{~cm}^{-1}$. The double peaks of the carboxylate vibration at $1587 \mathrm{~cm}^{-1}$ (in the range $1540-1650 \mathrm{~cm}^{-1}$, asymmetric) and $1403 \mathrm{~cm}^{-1}$ (in the range $1360-1450 \mathrm{~cm}^{-1}$, symmetric) in the spectra of CdSe particles show that the organics exist as carboxyl salts on the particle surface. The peak position of this doublet is similar to that observed for other mercaptocarboxylic salts.

The nonlinear optical characterization of the samples was carried out using the $Z$-scan technique at $532 \mathrm{~nm}[13,14]$. The samples to be investigated were translated through the focal point of a lens of focal length of $20 \mathrm{~cm}$. The beam waist radius $\omega_{0}$ was estimated to be $35.4 \mu \mathrm{m}$. The Rayleigh length $Z_{0}=$ $\pi \omega_{0}^{2} / \lambda$ was calculated to be $7.4 \mathrm{~mm}$. The samples were taken in a $1 \mathrm{~mm}$ cuvette which is much less than $Z_{0}$ which is an essential prerequisite for $Z$-scan experiment. The normalized transmittance for TPA in the open aperture condition is given by [15]

$$
T(Z, s=1)=\frac{1}{\sqrt{\pi q_{0}(Z, 0)}} \int_{-\infty}^{+\infty} \ln \left[1+q_{0}(Z, 0) e^{-\tau^{2}}\right] d \tau
$$




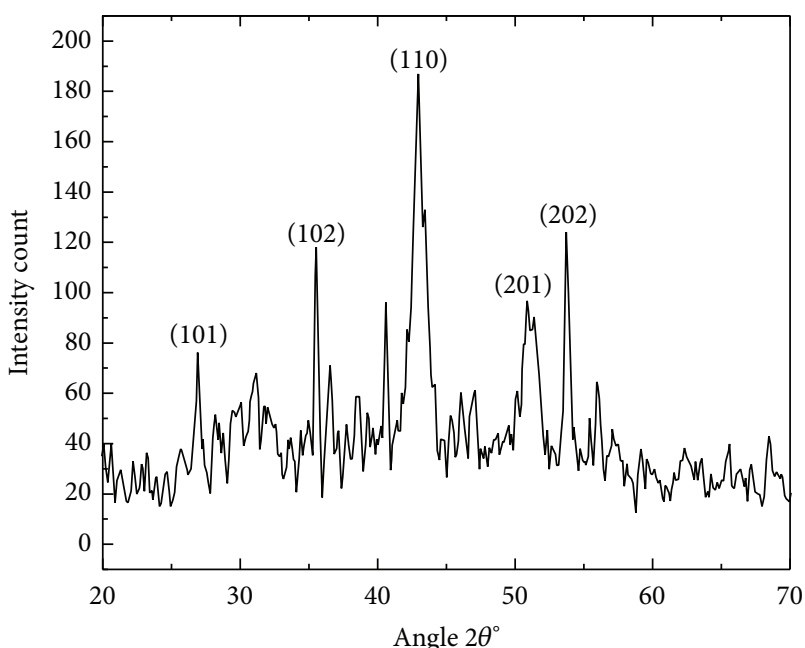

FIGURE 4: XRD spectra of $\mathrm{C}_{3}$ sample with the average radius of the particle being $4 \mathrm{~nm}$.

where

$$
q_{0}(Z, 0)=\frac{\beta I_{0} L_{\mathrm{eff}}}{\left(1+Z^{2} / Z_{0}^{2}\right)}
$$

The parameter $q_{0}$ is the depth of the open aperture $Z$-scan curve obtained from the theoretical fit and it is the measure of the intensity dependent absorption. Here, $I_{0}$ is the laser intensity in the focal plane, $\beta$ is the nonlinear optical absorption coefficient, and $L_{\text {eff }}$ is the effective thickness with linear absorption coefficient $\alpha$.

$L_{\text {eff }}$ is given by

$$
L_{\text {eff }}=\frac{\left(1-e^{-\alpha l}\right)}{\alpha} .
$$

The open-aperture curve exhibits a normalized transmittance valley at the focal point, indicating the presence of reverse saturable absorption (RSA) in the nanocolloidal solutions. The open aperture $Z$-scan plot for samples having different particle size is depicted in Figure 7. The fits of (2) to experimental data are shown as solid curves.

The data can be fitted well by assuming two-photon absorption (TPA) in the nonlinear optical absorption process. From this fit, we can confirm that the basic mechanism involved in the nonlinear absorption of nanocolloidal solutions of CdSe QDs is TPA process because the photon energy of the $532 \mathrm{~nm}$ laser is within the range $E_{g}<2 h \nu<2 E_{g}$, where $h \nu=2.33 \mathrm{eV}$ [15]. The nonlinear absorption coefficient $(\beta)$ calculated from the above fits shows a dependence on the particle size of the QDs. The experimental data show that $\beta$ value increases with increasing particle size. With a small decrease in the band gap, a significant increase in the nonlinear absorption is observed. In order to explain the optical nonlinearity, we mainly consider the band gap variation of CdSe QDs samples $\left(\mathrm{C}_{1}, \mathrm{C}_{2}\right.$, and $\left.\mathrm{C}_{3}\right)$.

$\mathrm{C}_{1}$ and $\mathrm{C}_{2}$ samples have band gap very near to $2.4(2.39 \pm$ $0.02)$ and $2.5(2.52 \pm 0.02)$, respectively, which is more than one-photon energy corresponding to $532 \mathrm{~nm}$. The data fitting shows that the nonlinear optical absorption is through twophoton absorption process in these samples. Our studies show that sample $\mathrm{C}_{3}$ has large optical nonlinearity $(2.47 \times$ $10^{-10} \mathrm{~m} / \mathrm{W}$ ) enhancement, compared to other samples as seen in Figure 7.

Band gap corresponding to this sample is $2.33 \mathrm{eV}$ to $2.33 \pm 0.02 \mathrm{eV}$ which is very near to the one-photon energy corresponding to $532 \mathrm{~nm}(2.33 \mathrm{eV})$. The fact that optical nonlinearity in this case is also due to the two-photon absorption reveals that two-photon excitation cross section is enhanced due to resonant one-photon absorption level. A small mismatch between band gap of sample $\mathrm{C}_{3}$ and one by photon energy will be compensated by phonon-assisted excitation. The enhancement also arises due to increased optical nonlinear interaction between the radiations and the particles. This can be explained by the fact that, with increase in particle size, there is an increase in the multiple scattering from the QDs. This increase in multiple scattering leads to large effective interaction length which in turn results in an enhancement in nonlinear absorption [14].

The open $Z$-scan scheme was also used to measure $\operatorname{Im} \chi^{(3)}$. The imaginary part of third-order susceptibility, $\operatorname{Im} \chi^{(3)}$, is related to $\beta$ through the following equation:

$$
\operatorname{Im} \chi^{(3)}=\frac{n_{0}^{2} C^{2} \beta}{240 \pi^{2} \omega}
$$

where $n_{0}=2.34$ is the linear refractive index of CdSe, $C$ is the velocity of light in vacuum, and $\omega$ is the angular frequency of the radiation used. The measured values of optical band gap for different particle size and nonlinear absorption coefficient at a wavelength of $532 \mathrm{~nm}$ for input laser power density of $0.54 \mathrm{GW} / \mathrm{cm}^{2}$ are given in Table 1 .

To study the optical limiting property of the sample, the nonlinear transmission of the sample is measured as a function of input fluence. Optical power limiting is effected through the nonlinear optical processes of the sample. An important term in the optical limiting study is the optical limiting threshold. Optical limiters are essentially those systems which transmit light at low input fluence or intensities but become opaque at high inputs. The optical limiting property is mainly found to be absorptive nonlinearity, which corresponds to the imaginary part of third-order susceptibility [16]. From the values of fluence at focus, the fluence values at other positions could be calculated using the standard equations for Gaussian beam waist. Such plots give a better comparison of the nonlinear absorption or transmission in the sample and are generated from $Z$-scan trace. Figure 8 illustrates the optical limiting response of the samples $\left(\mathrm{C}_{1}, \mathrm{C}_{2}\right.$, and $\left.\mathrm{C}_{3}\right)$.

The line in Figure 8 indicates the approximate fluence at which the normalized transmission begins to deviate from linearity. The fluence value corresponding to the onset of optical limiting (optical limiting threshold) is found to vary from $0.35 \mathrm{GW} / \mathrm{cm}^{2}$ to $0.572 \mathrm{GW} / \mathrm{cm}^{2}$ for the samples with input fluence of $0.54 \mathrm{GW} / \mathrm{cm}^{2}$. Our work shows that the semiconductor nanomaterial which has got a one-photon resonant absorption can be employed for third-order optical nonlinear material in which two-photon absorption will get 


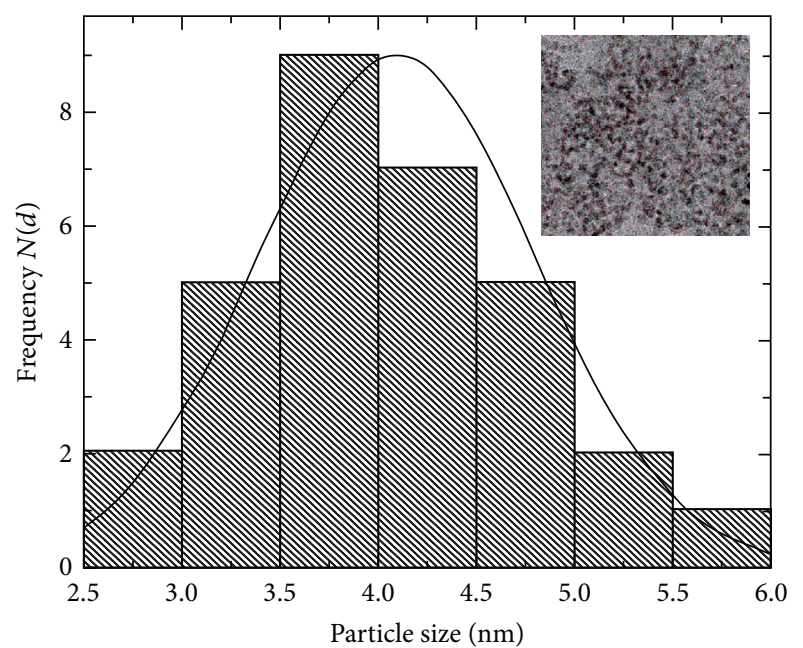

(a)

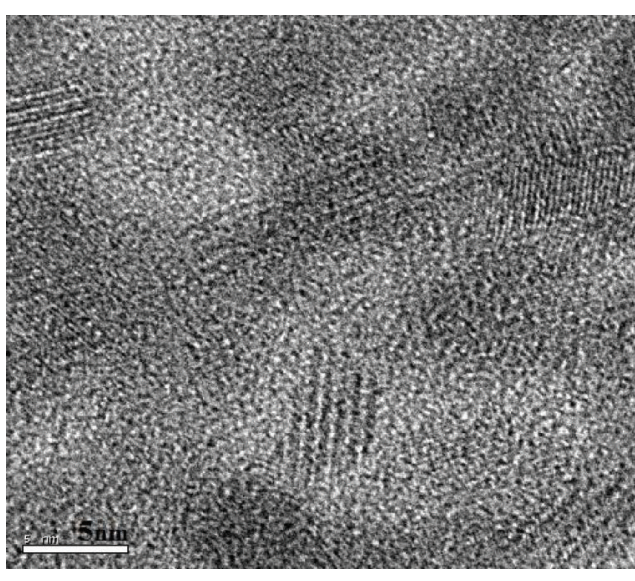

(b)

FIGURE 5: (a): Histogram and TEM image of $C_{3}$ sample with average particle size of $4.09 \mathrm{~nm}$. (b) HRTEM image of $\mathrm{C}_{3}$ sample.

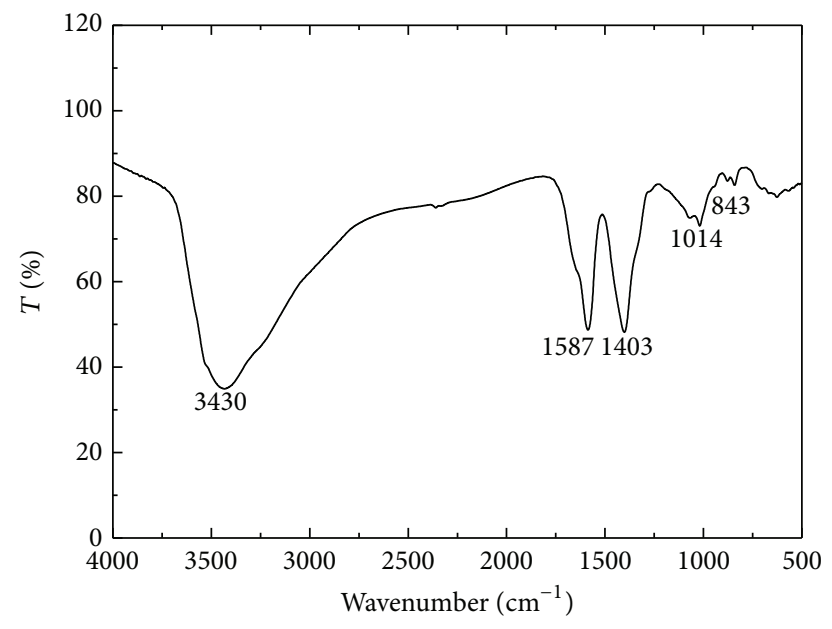

FIGURE 6: FTIR spectrum of the $\mathrm{C}_{3}$ sample.

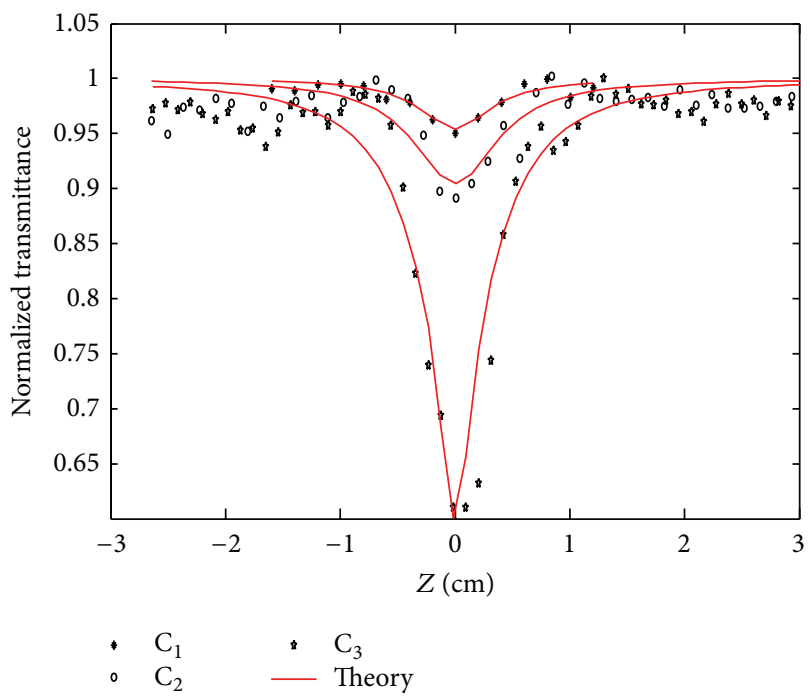

FIGURE 7: The open aperture $Z$-scan plot for different particle sizes of CdSe QDs $\left(\mathrm{C}_{1}, \mathrm{C}_{2}\right.$, and $\left.\mathrm{C}_{3}\right)$.

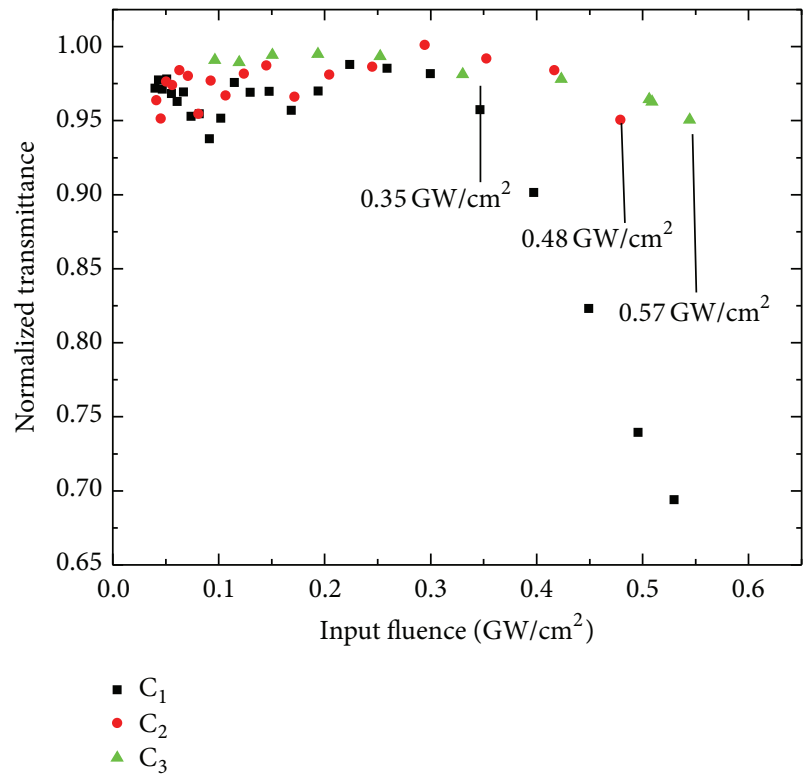

Figure 8: Optical limiting response of the samples $\left(\mathrm{C}_{1}, \mathrm{C}_{2}\right.$, and $\left.\mathrm{C}_{3}\right)$.

enhanced by the one-photon resonance energy level (Table 1). Such materials are useful as optical limiters with low threshold energy. Thus, it is clear from Figure 8 that the CdSe quantum dots with large particle size can be used for optical power limiting at high laser fluences. Thus, size of QDs has a significant effect on limiting performance.

\section{Conclusion}

To conclude, a simple synthetic route described in this paper permits the synthesis of high-quality CdSe QDs of different particle size in aqueous solution. The MSA-capped CdSe QDs possess a tuning effect in particle size and in band gap. Linear and nonlinear optical properties were studied using optical 
absorption, fluorescence spectroscopy, and open aperture $Z$ scan technique. The nonlinear absorption in the nanocolloidal solution can be attributed to the phenomenon of twophoton absorption. The measured $\beta$ values were found to enhance with decrease in optical energy gap. Our work shows that the semiconductor nanomaterial which has got a onephoton resonant absorption can be employed for third-order optical nonlinear material in which two-photon absorption will get enhanced by the one-photon resonance energy level.

\section{Conflict of Interests}

The authors declare that there is no conflict of interests regarding the publication of this paper.

\section{Acknowledgments}

The authors acknowledge DST for financial assistance and SAIF-STIC for XRD analysis.

\section{References}

[1] H. Fan, X. Wang, Q. Ren et al., "Investigation of the nonlinear absorption and optical limiting properties of two $[\mathrm{Q}]_{2}\left[\mathrm{Cu}\left(\mathrm{C}_{3} \mathrm{~S}_{5}\right)_{2}\right]$ compounds," Optics and Laser Technology, vol. 42, no. 5, pp. 732-736, 2010.

[2] I. Gerdova and A. Hache, "Third-order non-linear spectroscopy of CdSe and CdSe/ZnS core shell quantum dots," Optics Communications, vol. 246, no. 1-3, pp. 205-212, 2005.

[3] D. Maikhuri, S. P. Purohit, and K. C. Mathur, "Linear and nonlinear intraband optical properties of $\mathrm{ZnO}$ quantum dots embedded in $\mathrm{SiO}_{2}$ matrix," AIP Advances, vol. 2, no. 1, Article ID 012160, 2012.

[4] N. Venkatram, R. S. S. Kumar, and D. Narayana Rao, "Nonlinear absorption and scattering properties of cadmium sulphide nanocrystals with its application as a potential optical limiter," Journal of Applied Physics, vol. 100, no. 7, Article ID 074309, 2006.

[5] R. W. Meulenberg, J. R. I. Lee, A. Wolcott, J. Z. Zhang, L. J. Terminello, and T. van Buuren, "Determination of the exciton binding energy in CdSe quantum dots," ACS Nano, vol. 3, no. 2, pp. 325-330, 2009.

[6] A. Patra, N. Venkatram, D. Narayana Rao, and T. P. Radhakrishnan, "Optical limiting in organic molecular nano/microcrystals: nonlinear optical effects dependent on size distribution," Journal of Physical Chemistry C, vol. 112, no. 42, pp. 16269-16274, 2008.

[7] W. Sun, C. M. Lawson, G. M. Gray, C. Zhan, and D. Wang, "Degenerate four-wave mixing and Z-scan measurements of stilbazolium derivatives," Applied Physics Letters, vol. 78, no. 13, pp. 1817-1819, 2001.

[8] S. Mathew, A. D. Saran, B. Singh Bhardwaj et al., "Size dependent optical properties of the CdSe-CdS core-shell quantum dots in the strong confinement regime," Journal of Applied Physics, vol. 111, no. 7, Article ID 074312, 2012.

[9] X. Chen, J. L. Hutchison, P. J. Dobson, and G. Wakefield, "Highly luminescent monodisperse CdSe nanoparticles synthesized in aqueous solution," Journal of Materials Science, vol. 44, no. 1, pp. 285-292, 2009.
[10] B. Su, D. J. Fermin, J. P. Abid, N. Eugster, and H. H. Girault, "Adsorption and photoreactivity of CdSe nanoparticles at liquid-liquid interfaces," Journal of Electroanalytical Chemistry, vol. 583, no. 2, pp. 241-247, 2005.

[11] A. L. Rogach, A. Kornowski, M. Gao, A. Eychmüller, and H. Weller, "Synthesis and characterization of a size series of extremely small thiol-stabilized CdSe nanocrystals," The Journal of Physical Chemistry B, vol. 103, no. 16, pp. 3065-3069, 1999.

[12] L. Brus, "Zero-dimensional "excitons" in semiconductor clusters," IEEE Journal of Quantum Electronics, vol. 22, no. 9, pp. 1909-1914, 1986.

[13] A. Thankappan, S. Thomas, and V. P. N. Nampoori, "Effect of betanin natural dye extracted from red beet root on the non linear optical properties $\mathrm{ZnO}$ nanoplates embedded in polymeric matrices," Journal of Applied Physics, vol. 112, no. 12, Article ID 123104, 2012.

[14] I. Sebastian, S. Divya, V. P. N. Nampoori, P. Radhakrishnan, and S. Thomas, "Impact of intermediate localized states on nonlinear optical absorption of Ga-Ge-Se nanocolloidal solutions," Applied Physics Letters, vol. 102, no. 3, Article ID 031115, 2013.

[15] M. Sheik-Bahae, A. A. Said, T.-H. Wei, D. J. Hagan, and E. W. Van Stryland, "Sensitive measurement of optical nonlinearities using a single beam," IEEE Journal of Quantum Electronics, vol. 26, no. 4, pp. 760-769, 1990.

[16] F. M. Qureshi, S. J. Martin, X. Long et al., "Optical limiting properties of a zinc porphyrin polymer and its dimer and monomer model compounds," Chemical Physics, vol. 231, no. 1, pp. 87-94, 1998. 

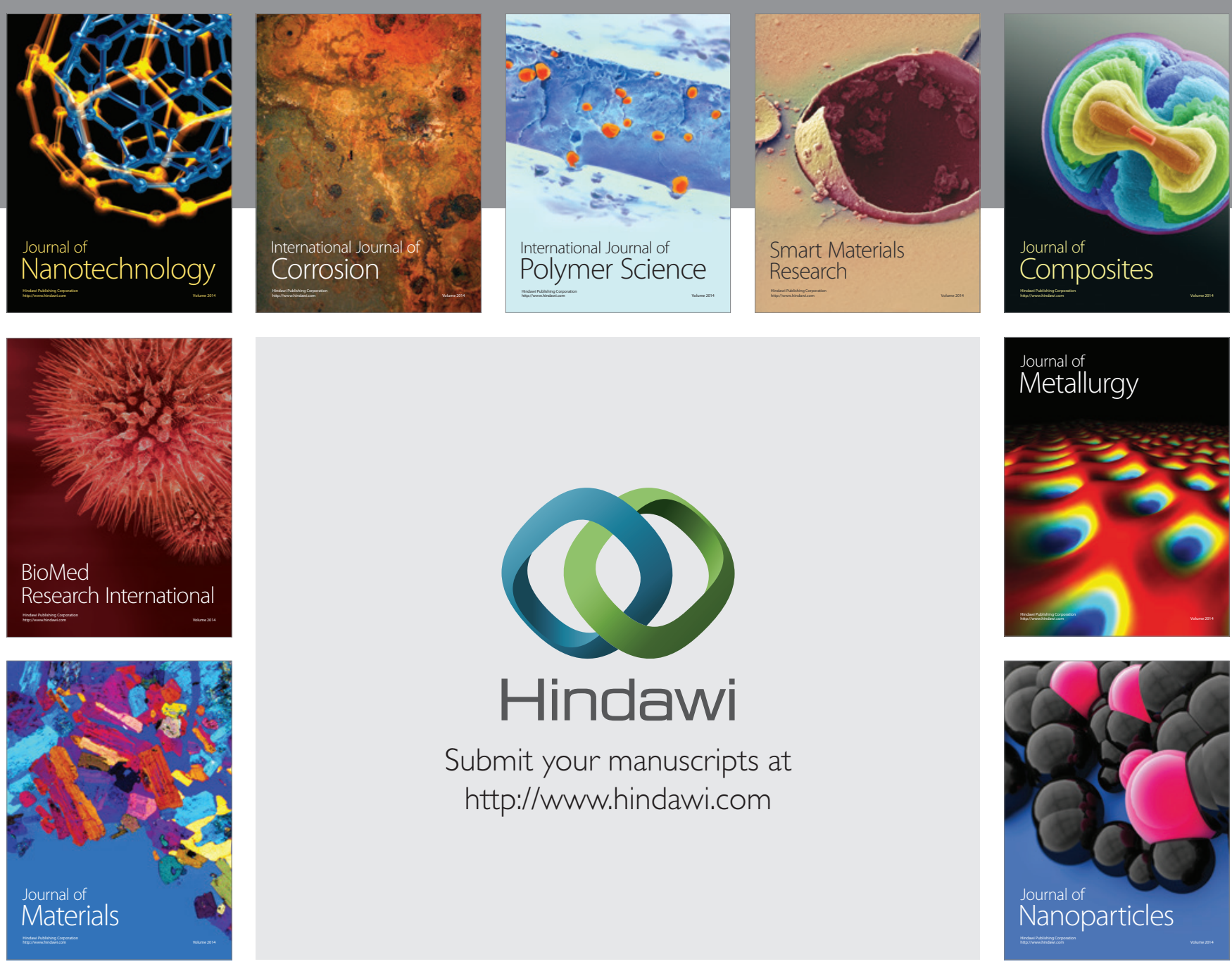

Submit your manuscripts at http://www.hindawi.com
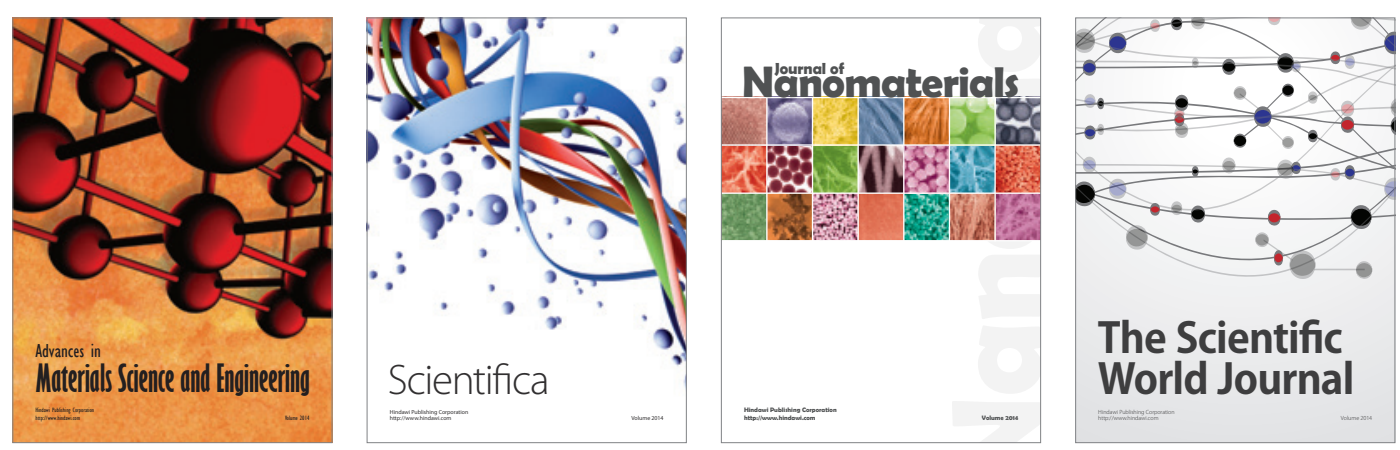

\section{The Scientific World Journal}
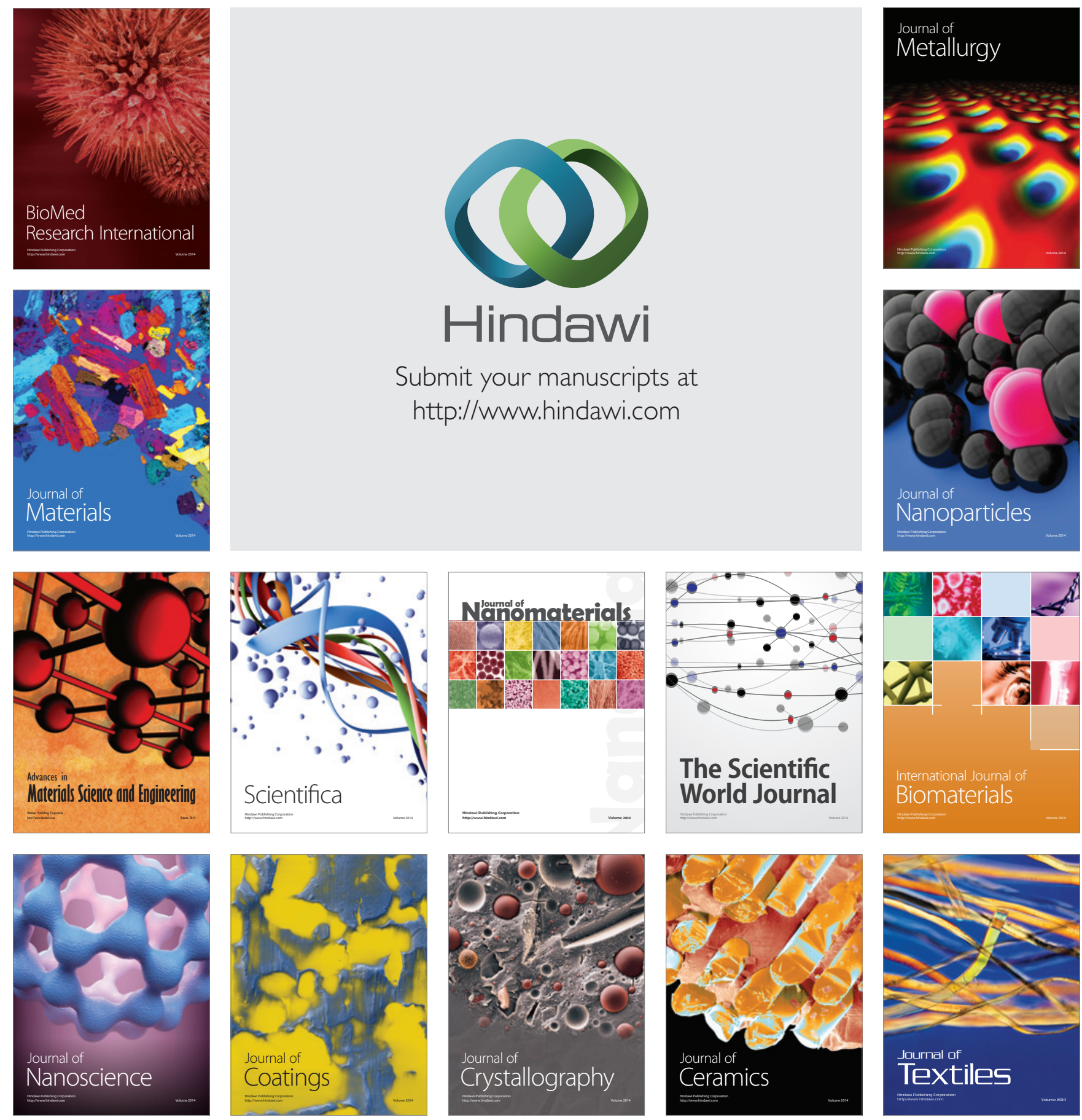